\title{
Ifglfl Monatssehrift
}

für

\section{Psychiatrie und Neurologie.}

Herausgegeben von

Th. Ziehen. Band XXVIII.

Mit zahlreichen Abbildungen im Text und 20 Tafeln.

; $\quad$ S.\# K. '

BERL I N 1910

VERLAG VON S. KARGER

KARLSTRASSE lä. 
Alle Rechte vorbehalten..

Gedruckt bei Imberg \& Lefson G. m. b. H. in Berlin STT. GS. 


\title{
Inhalts -Verzeichnis.
}

\author{
Original-Arbeiten.
}

Alessandrini, Paul, Die Anencephalie vom anatomo-embryologischen, physiologischen und pathogenetischen Stand punkt betrachtet. Ein Beitrag zur Lehre von der inneren Sekretion. (Hierzu Taf. XV-XVII)...

Auerbach, Siegm., Ueber einen Fall von akuter absteigender

Paralyse.

Bechterew, W. v., Ueber das manisch-melancholische Irresein.

Zur Frage der Beziehung zwischen manischen und

melancholischen Zuständen

Behr, H., Zur Histopathologie der juvenilen Form der fa

miliären amaurotischen Idiotie

Bickel, Heinrich, Zur Anatomie und Aetiologie der Dementia

praecox

Bisgaard, A., Methode zu objektiver Schätzung der so

genannten Phase I bei Untersuchungen des Liquor cere

brospinalis

Bresowshy, M., Ueber die Beziehungen der Paranoia acuta

hallucinatoria (Westphal) zur Amentia (Meynert) . 46, 134

Eiders, C, Die motorischen Centren und die Form des Vorder-horns in

den fünf letzten Segmenten des Cervikalmarkes und dem ersten

Dorsalsegmente eines Mannes, der ohne linken Vorderarm geboren ist.

(Hierzu Taf. XIX-XX) . 491

Fabritius, H., Versuch einer Psychophysiologie des Gefühls 400

Ferrari, Manlio, Histologische Untersuchungen am Zentral

nervensystem von Abkömmlingen chronisch alkoholi

sierter Tiere. (Hierzu Taf. XVIII.)

Franke, Georg, Klinischer Beitrag zum Krankheitsbilde der

transkortikalen motorischen Aphasie

Fröderström, $H$., und V. Wigert, Ueber das Verhältnis der

Wassermannschen Reaktion zu den zytologischen und

chemischen Untersuchungsmethoden der Spinalflüssig

keit

Gregor, A., Beiträge zur Psychologie der Aussage von Geistes

kranken. I. Progressive Paralyse ............................................290, 428

Klieneberger, O. L., Ein eigentümlicher Liquorbefund bei

Rückenmarkstumoren

Liebscher, Carl, Ueber Sehstörungen nervösen Ursprungs

(Erg.-Heft)

Lilienstein, S., Ueber Irrenanstalten im Orient und in

Australien. 
Maas, Otto, Beitrag zur Kenntnis der Recklinghausenschen

Krankheit. (Hierzu Taf. V-VIII.) (Erg.-Heft) ... 167

Mailing, Knud, Tabes dorsalis juvenilis mit Autopsie. (Hierzu

Taf. XIII-XIV)

Moerchen, Friedrich, Epileptolde und delirante Zustände bei

kombiniertem Morphium- und Isopralmissbrauch ... 35

Müller, E. Herrn., Einige Beziehungen des Alkoholismus zur

Aetiologie der Epilepsie. (Erg.-Heft)....

Münzer, E., und $H$. Wiener, Experimentelle Beiträge zur

Lehre von den endogenen Easersystemen des Rücken

markes

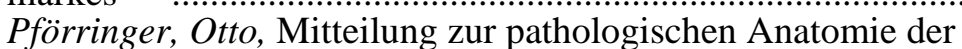

Pachymeningitis cervicaifa hypertrophica ..... 120

Pförtner, 0., Die körperlichen Symptome beim Jugendirresein 208

Sano, Torata, Beitrag zur vergleichenden Anatomie der

Substantia nigra, des Corpus Luysii und der Zona incerta

(Hierzu Taf. I-II.) (Fortsetzung)

,-- - Fortsetzung. (Hierzu Taf. IX $-\mathrm{X}$.)

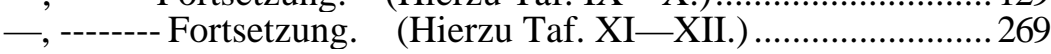

—, ------- Schluss ............................................................... 367

Seige, Max, Die Aufbrauchtheorie Edingers in ihrer An

wendung auf die Dementia paralytica 109

Ulrich, Martha, Beiträge zur Kenntnis der Stäbchenzellen im

Zentralnervensystem. (Hierzu Taf. HJ.) (Erg.-Heft). 24

Walter, F. K., Studien über den Liquor cerebrospinalis.

(Hierzu Taf. IV.) (Erg.-Heft).

Wendenburg, K., Ueber Chorea infectiosa und Chorea

hysterica

\section{Berichte.}

27. Kongress für innere Medizin in Wiesbaden vom 18.--21.

April 1910. Von Dr. 8. Lilienstein in Bad Nauheim . . 7657. Versammlung mittelrheinischer Aerzte in Frankfurt a. M.

am 22. Mai 1910. Von Dr. 8. Lilienstein in Bad Nauheim 7835. Wander-Versammlung der südwestdeutschen Neurologen und Irrenärzte am 28. und 29. Mai 1910. Von Dr. 8.

Lilienstein in Bad Nauheim.

IV. Internationaler Kongress zur Fürsorge für Geisteskranke in Berlin, 3.-7. Oktober 1910. Bericht, erstattet von

Dr. S. Lilienstein in Bad Nauheim. 473, 539

4. Jahresversammlung der Gesellschaft deutscher Nervenärzte in Berlin vom 6.-8. Oktober 1910. Bericht, erstattet von Dr. 8. Lilienstein in Bad Nauheim.

Buchanzeigen $91,187,278,376,481,570$

Tagesgeschichtliches und Personalien 94, 188, 281, 376, 482, 572 